\title{
Sex Differences and Similarities in Hippocampal Cellular Proliferation and the Number of Immature Neurons during Adolescence in Rats
}

\author{
Alina Siddiqui Russell D. Romeo \\ Department of Psychology and Neuroscience and Behavior Program Barnard College of Columbia University, \\ New York, NY, USA
}

\section{Keywords}

Adolescence · Dentate gyrus · Hippocampus .

Neurogenesis · Puberty

\begin{abstract}
Adolescence is associated with significant reductions in hippocampal cellular proliferation and neurogenesis, the physiological and behavioral implications of which are unclear. Though sex differences exist in these proliferative processes in adulthood, relatively little is known about the role sex plays in these adolescent-related changes. To address this gap, we examined cross-sectional area of the dentate gyrus and cellular proliferation, as measured by Ki-67 immunohistochemistry, in pre- (30 days), mid- (45 days), and post-adolescent (70 days) male and female rats. We also investigated the number of immature neurons using doublecortin (DCX) immunohistochemistry in pre- and post-adolescent males and females. Despite increases in the size of the dentate gyrus during adolescence, we found significant adolescent-related decreases in hippocampal proliferation in both males and females, with a more dramatic decrease in males, indicating both age- and sex-dependent changes in the dentate gyrus. We also found an adolescent-related decline in the number of immature neurons in the dentate gyrus of male rats and a female-biased sex difference in the number of immature neurons in adults. Given these significant changes in the dentate gyrus, these data suggest that this period in de-
\end{abstract}

\section{KARGER}

(c) 2019 S. Karger AG, Basel

E-Mail karger@karger.com

www.karger.com/dne velopment might be particularly sensitive to internal and external factors known to modulate neurogenesis, with potential sex-specific neurobehavioral ramifications.

(c) 2019 S. Karger AG, Basel

\section{Introduction}

Adolescent maturation is associated with many structural and functional changes in the brain, particularly within cortical and limbic regions [1-3]. One such change is in the number of newly born cells in the dentate gyrus of the hippocampal formation. For instance, studies in rats and mice have indicated a substantial decline in cellular proliferation in this structure that occurs at pubertal onset and is maintained into adulthood [4-11]. The implications of these changes during this developmental stage are unclear but might contribute to the significant shifts in neurobehavioral functions noted during adolescence [12].

In addition to development, sex of the individual affects cellular proliferation and neurogenesis in the hippocampal formation [13]. Adult female rats have higher levels of cellular proliferation in the dentate than males [14], and gonadal hormones can further modulate the difference in the birth and survival of newly born cells [15]. Specifically, ovarian hormones in females, such as estradiol and progesterone, promote both cellular prolif- 
eration and survival $[14,16]$, while testicular hormones in males, such as testosterone, support cell survival [17]. Though levels of gonadal hormones substantially change during pubertal maturation [18], the adolescent-related decrease in hippocampal cell proliferation occurs independent of these hormonal changes, at least in males [10]. That is, males castrated prior to puberty continue to show the decline in both cellular proliferation and neurogenesis into adulthood [10].

It is currently unknown whether there are sex differences in cellular proliferation or the number of immature neurons in the peripubertal dentate gyrus. It has been reported that neonatal males have greater cellular proliferation in the dentate gyrus than females $[19,20]$, so it is possible that these sex differences persist into the postweanling, pubertal stages of development. Furthermore, as changes in cortical and limbic gray matter often display curvilinear patterns [1], with differences between males and females [21-23], the decline in cellular proliferation from pubertal onset to adulthood might also display similar nonlinear patterns with unique differences between the sexes.

To bridge this gap in our understanding of adolescentand sex-dependent changes in cellular proliferation and the number of immature neurons in the hippocampal formation, we examined these parameters in the dentate gyrus of both male and female rats before, during, and after adolescent development. We hypothesized that there would be adolescent-related decreases in the number of proliferating cells and immature neurons, with significant differences between males and females.

\section{Materials and Methods}

\section{Animals}

Male and female Sprague-Dawley rats were obtained from our breeding colony at Barnard College. All animals were weaned at 21 days of age and housed 2 per cage in clear polycarbonate cages $(45 \times 25 \times 20 \mathrm{~cm})$ with wood chip bedding. All animals were maintained on a 12-h light-dark schedule (lights on at $09.00 \mathrm{~h}$ ). Animals had ad libitum access to food and water, and the animal room was maintained at $21 \pm 2{ }^{\circ} \mathrm{C}$. All procedures were carried out in accordance with the guidelines established by the National Institutes of Health Guide for the Care and Use of Laboratory Animals and approved by the Institutional Animal Care and Use Committee of Columbia University.

\section{Perfusion and Tissue Collection}

Rats were perfused at 30 (pre-adolescent), 45 (mid-adolescent), or 70 (post-adolescent) days of age with heparinized saline rinse and $4 \%$ paraformaldehyde ( $n=6-8$ per age and sex). Prior to perfusion, animals were weighed and deeply anesthetized with ket- amine (80 mg/kg, i.p.) and xylazine (5 mg/kg, i.p.). Brains were post-fixed for $24 \mathrm{~h}$ in $4 \%$ paraformaldehyde in $0.1 \mathrm{M}$ phosphate buffer (PB) and then incubated in $20 \%$ sucrose in $0.1 \mathrm{M} \mathrm{PB}$ at $4{ }^{\circ} \mathrm{C}$. Brains were sectioned coronally in 40 - $\mu \mathrm{m}$-thick sections into 4 serial sets on a cryostat and stored in cryoprotectant (1:1 of $20 \%$ sucrose in $0.1 \mathrm{M} \mathrm{PB}$ and ethylene glycol) at $-20^{\circ} \mathrm{C}$ until processed for histology (see below).

\section{Cross-Sectional Area Measurement of Dentate Gyrus}

To assess possible effects of age and sex on the cross-sectional area of the dentate gyrus, the first set of sections for each animal were Nissl stained. These sections were adjacent to the sections used for Ki-67 immunohistochemistry (see below), separated by $120 \mu \mathrm{m}$, and anatomically matched across subjects (corresponding to plates 56-62 in a standard rat atlas [24]). Measurements were made using ImageJ (Wayne Rasband, National Institutes of Health, Bethesda, MD, USA) from images captured under a $2.5 \times$ objective. The cross-sectional area of each dentate gyrus was measured from each section, averaged for each animal, and presented as the average cross-sectional area $\left(\mathrm{mm}^{2}\right)$.

\section{Ki-67 and DCX Immunohistochemistry}

For immunohistochemistry, the second (Ki-67) and third (DCX) set of sections were washed in $0.1 \mathrm{M} \mathrm{PB}$ and incubated for $10 \mathrm{~min}$ in $0.05 \% \mathrm{H}_{2} \mathrm{O}_{2}$ in $0.1 \mathrm{M} \mathrm{PB}$ saline (PBS). Sections were then washed in $0.1 \mathrm{M} \mathrm{PB}$ with $0.1 \%$ Triton-X-100 (PBT), blocked for $1 \mathrm{~h}$ in $2 \%$ normal goat serum in PBT, and then incubated in either rabbit anti-Ki-67 (1:1,000; AB15580; Abcam, Cambridge, MA, USA) or guinea pig anti-DCX (1:10,000; AB2253; Millipore Sigma, Burlington, MA, USA) in normal goat serum block for $24 \mathrm{~h}$ at $4{ }^{\circ} \mathrm{C}$. The tissue was washed in PBT and incubated for $1 \mathrm{~h}$ in either goat anti-rabbit or anti-guinea pig secondary antibodies (1: 200; Vector Laboratories) in PBT at room temperature and then incubated in Avidin-Biotin Complex at room temperature (1:250; Vectastain ABC Kit, Vector Laboratories) for $1 \mathrm{~h}$. The sections were then washed in PBS and incubated for $5 \mathrm{~min}$ in 3,3'-diaminobenzidine in a $3 \mathrm{M}$ sodium acetate buffer containing $0.05 \%$ $\mathrm{H}_{2} \mathrm{O}_{2}$. For Ki-67 immunostaining, the 3,3'-diaminobenzidine was nickel enhanced, while no nickel enhancement was used for DCX immunostaining. Tissue was then washed in PBS, mounted on Fisher Brand Plus slides (Fischer Scientific, Pittsburg, PA, USA), dried, dehydrated in 70, 95, and 100\% ethanol, cleared in xylenes, and coverslipped with DPX Mountant (Sigma-Aldrich, St Louis, MO, USA).

\section{Microscopy and Quantification}

Six or three anatomically matched sections from the dentate gyrus, separated by $120 \mu \mathrm{m}$, were used to measure Ki-67- and DCX-positive cells, respectively (corresponding to plates 56-62 or 58-60, respectively; [24]). For Ki-67 analysis in the dentate, cells were counted under a $40 \times$ objective across the entire dentate gyrus of each section and averaged. As we also measured the cross-sectional area of each dentate gyrus, we present these data as average number of Ki-67-positive cells per unit area $\left(\mathrm{mm}^{2}\right)$ of the dentate gyrus. For DCX analysis in the dentate, cell counts were made under a $40 \times$ objective in the dentate gyrus by placing a grid of $10,000 \mu \mathrm{m}^{2}$ superimposed on the images. Bilateral counts from the upper and lower blades of the dentate gyrus were made for each brain section and averaged. Data are presented as the estimated average number of DCX-positive cells per $\mathrm{mm}^{2}$. It is important to 


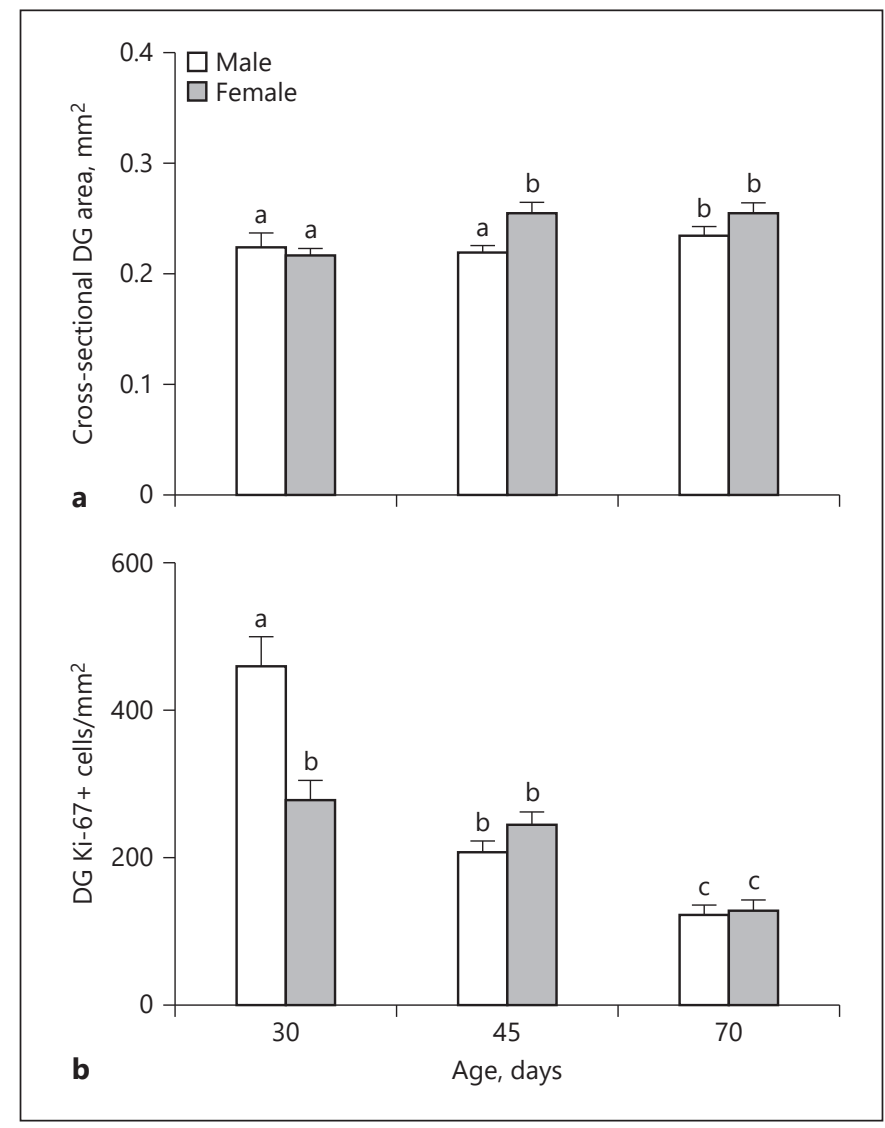

Fig. 1. Mean ( \pm SEM) cross-sectional area $\left(\mathrm{mm}^{2} ; \mathbf{a}\right)$ and number of Ki-67-positive cells per $\mathrm{mm}^{2}(\mathbf{b})$ in the dentate gyrus of preadolescent (30 days of age), mid-adolescent (45 days of age), and post-adolescent (70 days of age) male (white bars) and female (grey bars) rats. Bars that share a letter are not significantly different from one another.

note that only the animals in the 30 and 70 days of age groups were processed for DCX immunohistochemistry, as the greatest change in cellular proliferation was observed between these 2 ages.

\section{Statistical Analysis}

Two-way ANOVAs (sex $\times$ age) were used for statistical analyses, and significant effects were further analyzed with Tukey's honestly significant difference post hoc tests. Data are reported as the mean \pm SEM, and differences were considered significant at $p<$ 0.05. All statistical analyses were performed using GraphPad PRISM, version 5.04 (GraphPad Software Inc., San Diego, CA, USA).

\section{Results}

For the cross-sectional area of the dentate gyrus, 2-way ANOVAs revealed a significant interaction between age and sex, such that there is an increase in the area of the dentate in both males and females as they progress through adolescence, but this increase occurs earlier in females $(F[2,40]=6.14, p<0.05$; Fig. 1a). Specifically, the cross-sectional area of the female dentate gyrus increases between 30 and 45 days of age, while the cross-sectional area in males increases only after 45 days of age.

A 2-way ANOVA also revealed a significant interaction between age and sex in the context of Ki-67-positive cell number in the dentate gyrus $(F[2,40]=16.62, p<$ 0.05 ; Fig. 1b). On this measure, pre-adolescent males had significantly more Ki-67-positive cells than pre-adolescent females, and both males and females demonstrated a decline in Ki-67-positive cell number from 30 to 70 days of age. No sex differences were observed in overall cell proliferation at 45 or 70 days of age, indicating the preadolescent to post-adolescent decrease in cellular proliferation in the dentate is greater in males compared to females. In fact, the decrease in males reaches approximately $75 \%$, while in females the decrease is about $55 \%$. Figure 2 shows representative photomicrographs of these differences in the number of Ki-67 cells in dentate gyrus of preand post-adolescent males and females (Fig. 2).

To determine if changes in proliferation are paralleled by changes in the number of immature neurons, we conducted a 2-way ANOVA on the number DCX-positive cells in the dentate between pre-adolescent and post-adolescent males and females and found a significant interaction between age and sex $(F[1,20]=5.61, p<0.05$; Fig. 3). Specifically, there was a significant decline in DCX-positive cell number in males from 30 to 70 days of age, while 70-day-old females had significantly more DCX-positive cells than 70-day-old males.

\section{Discussion}

The present study demonstrates that there are significant age- and sex-related changes in cellular proliferation and the number of immature neurons in the dentate gyrus of the hippocampal formation throughout adolescence. Specifically, pre-adolescent males show significantly more cellular proliferation than pre-adolescent females, but these levels of proliferation are equal by the time both males and females reach young adulthood. Males also had a pubertal decline in the number of immature neurons, paralleling the observed reduction in proliferation. In comparison, females show no pubertal decline in the number of young neurons and adult females had more immature neurons than adult males. 

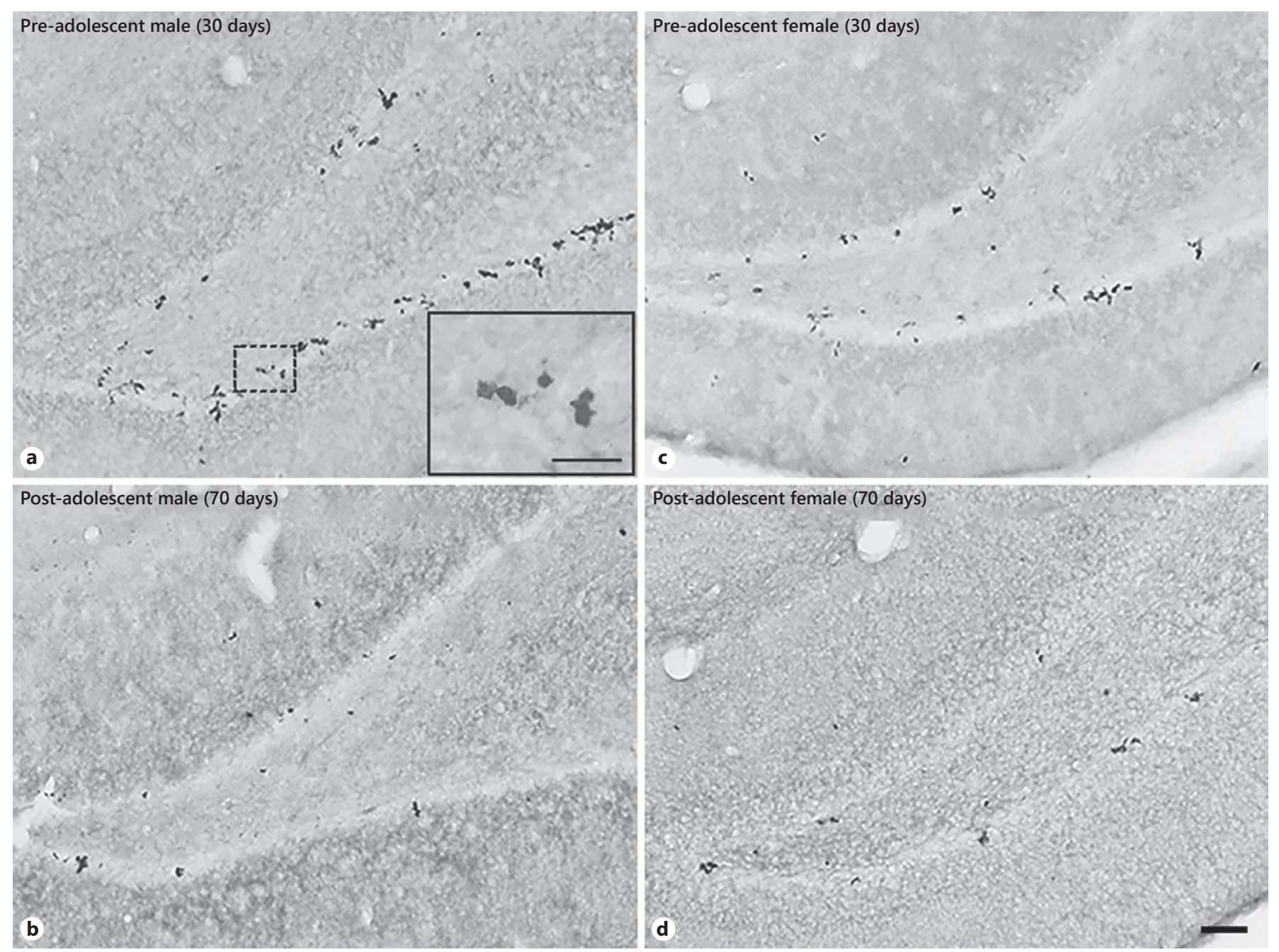

Fig. 2. Representative photomicrographs of Ki-67-positive cells in the dentate gyrus of a pre-adolescent (30 days of age) male (a) and female (c) and post-adolescent (70 days of age) male (b) and female

(d) rats. Scale bar $=50 \mu \mathrm{m}$. The inset in panel $\mathrm{A}$ is a higher magnification picture of $6 \mathrm{Ki}-67$-positive cells (scale bar $=25 \mu \mathrm{m}$ ) from the area in the box with hashed lines.

Though the implications of these neurobiological changes are currently unknown, they might contribute to the significant physiological and behavioral alterations reported to occur during adolescence, which often show sex differences, such as regulation of hormonal stress reactivity and associative memory functions $[25,26]$.

Previous studies have reported sex differences in hippocampal cell proliferation shortly after birth, such that neonatal male rats have more proliferation, as measured by either Ki-67 or BrdU immunohistochemistry, in the dentate gyrus than neonatal females $[19,20]$. The present study suggests that this sex difference is maintained, at least until the onset of puberty, as the pre-adolescent males have about $40 \%$ more proliferating cells than the

pre-adolescent females. Though it is unclear what purpose this difference in proliferation serves the developing animal, these data indicate that this considerable sex difference in proliferation is eliminated by time adolescent development is complete, with no differences in proliferation between our 70-day-old, post-adolescent males and females. It should be noted that an earlier study did find a female-biased sex difference in cellular proliferation in the adult dentate gyrus [14], which is different from the current results. However, this earlier study used short-term exposures to the thymidine analog, BrdU, to label proliferating cells [14], while we used Ki-67 as a marker of proliferation. Thus, methodological differences between these studies may account for these discrepant 


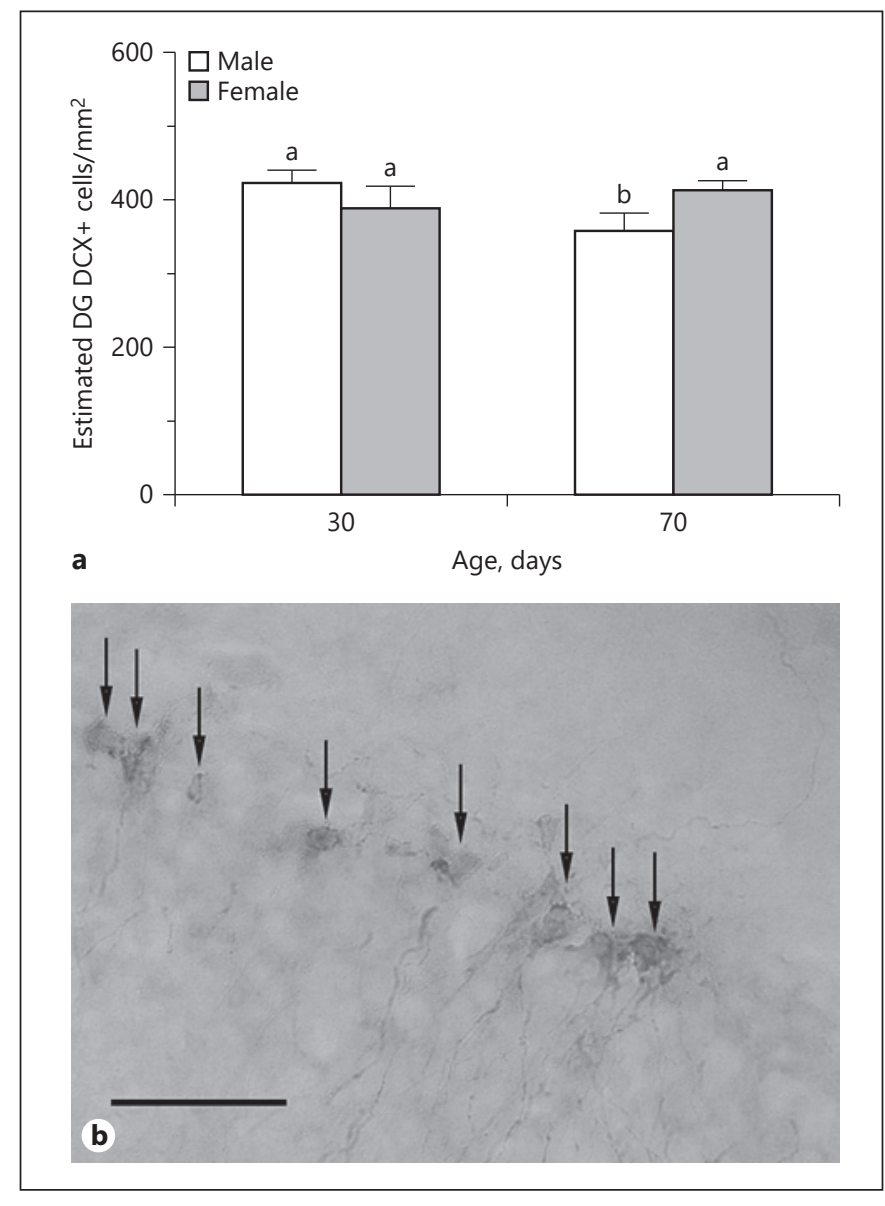

Fig. 3. Mean ( \pm SEM) estimated number of DCX-positive cells per $\mathrm{mm}^{2}$ in the dentate gyrus of pre-adolescent (30 days of age) and post-adolescent (70 days of age) male (white bars) and female (grey bars) rats (a). In (a), bars that share a letter are not significantly different from one another. $\mathbf{b}$ Is a representative photomicrograph of DCX-positive cells (black arrows) on a portion of the lower blade in the dentate gyrus of an adult female rat (b). Bar $=50 \mu \mathrm{m}$.

results. Regardless, this body of work suggests that sex differences in the rate of hippocampal cellular proliferation during earlier neonatal and peri-adolescent stages of development are significantly attenuated by the time reproductive maturity is attained.

The findings that the decline in proliferation is paralleled by a decrease in immature neurons in male rats from preadolescence into adulthood are consistent with previous studies $[10,27]$. Interestingly, however, a similar decline in immature neurons was not found in the females across the adolescent period, despite the noted reduction in cellular proliferation. These data suggest that proliferating cells in males are following a different maturational path than those in females during adolescence. While we quantified prolif- eration levels and the number of immature neurons, we did not measure survival of newly born cells nor did we examine the possibility that proliferating cells are differentiating into nonneuronal cell types, such as glia. No differences in cell survival have been found between adult males and females [14], but it is possible that more newly born cells in adult females differentiate into neurons, while more proliferating cells in adult males differentiate into glial cells. Along these lines, pubertal development has been shown to affect the number and morphology of microglia [28] and gonadal hormones are known to modulate the morphology of astrocytes in males and females [29, 30], suggesting a potential role for glial elements contributing to these observed changes. Another possibility that could account for these different results is that these maturational events might occur earlier in females than males. In fact, our data on the area of the dentate gyrus indicate that some of these adolescent-related changes arise sooner in females. Therefore, significant decreases in both cellular proliferation and the number of newly born neurons may begin earlier than 30 days of age in females. Though these possibilities are not mutually exclusive, future studies quantifying both neurogenesis and gliogenesis across the peri-adolescent period and into adulthood will be needed to determine potential maturational differences and the ultimate fate of these proliferating cells in the adolescent dentate gyrus of both males and females.

Contrary to the results of the current study, a previous experiment comparing the density of DCX-positive cells in the dentate gyrus of adult rats did not find a significant difference between males and females [31]. This inconsistency might be due to the differences in the age of the experimental subjects used, as the adult rats in the present study were significantly younger (i.e., 70 days of age) than the 4-monthold animals that were used in this previous study where no sex differences were observed [31]. If this were the case, these data would suggest that there are further sex-dependent changes in the number of proliferating cells and immature neurons in the dentate gyrus after young adulthood.

Cross-sectional area of dentate was found to increase significantly in both males and females, with the increase in females occurring earlier than males. These data support previous studies in male rats that found increases in the area of the dentate gyrus from the adolescent stage of development to adulthood [10,32]. This increase in the area of dentate gyrus in both males and females is paradoxical given the significant decrease in cellular proliferation happening in both sexes during this time. However, several factors might mediate this increase in area of the dentate gyrus that are independent of changes in the number of cells, such as changes in dendritic morphology and somal size of gran- 
ule neurons in the dentate gyrus, factors known to increase during neonatal and pubertal development [33].

In summary, these data indicate significant changes in the number of proliferating cells and immature neurons occur during adolescence within the dentate gyrus of rats, with unique developmental trajectories in males and females. While sex differences in hippocampal structure, function, and development are well-established [34], few studies have examined the impact of pubertal and adolescent development on hippocampal proliferation and neurogenesis. As newly born cells in the hippocampal formation are vital to cognitive functions, stress resilience, and memory formation [35-37], it will be important for future investigation to assess whether disrupting proliferation and/or neurogenesis during this dynamic stage of dentate gyrus development contribute to the neurobehavioral vulnerabilities that often emerge during adolescence.

\section{Acknowledgment}

We would like to thank Page Buchanan for excellent animal care.

\section{Statement of Ethics}

All procedures were carried out in accordance with the guidelines established by the National Institutes of Health Guide for the Care and Use of Laboratory Animals and approved by the Institutional Animal Care and Use Committee of Columbia University.

\section{Disclosure Statement}

The authors have no conflicts of interest to declare.

\section{Funding Sources}

This work was supported in part from a grant from the National Science Foundation IOS-1456577 (to R.D.R.).

\section{Author Contributions}

A.S. and R.D.R.: designed and conducted these experiments and both authors contributed to the writing and editing of the manuscript.

\section{References}

1 Giedd JN, Raznahan A, Alexander-Bloch A, Schmitt E, Gogtay N, Rapoport JL. Child psychiatry branch of the National Institute of Mental Health longitudinal structural magnetic resonance imaging study of human brain development. Neuropsychopharmacology. 2015 Jan;40(1):43-9.

2 Juraska JM, Sisk CL, DonCarlos LL. Sexual differentiation of the adolescent rodent brain: hormonal influences and developmental mechanisms. Horm Behav. 2013 Jul;64(2): 203-10.

3 Spear LP. Adolescent neurodevelopment. J Adolesc Health. 2013 Feb;52(2 Suppl 2):S713.

4 He J, Crews FT. Neurogenesis decreases during brain maturation from adolescence to adulthood. Pharmacol Biochem Behav. 2007 Feb;86(2):327-33.

5 Heine VM, Maslam S, Joëls M, Lucassen PJ. Prominent decline of newborn cell proliferation, differentiation, and apoptosis in the aging dentate gyrus, in absence of an age-related hypothalamus-pituitary-adrenal axis activation. Neurobiol Aging. 2004 Mar;25(3):361-75.

6 Kim YP, Kim H, Shin MS, Chang HK, Jang $\mathrm{MH}$, Shin MC, et al. Age-dependence of the effect of treadmill exercise on cell proliferation in the dentate gyrus of rats. Neurosci Lett. 2004 Jan;355(1-2):152-4.

7 McDonald HY, Wojtowicz JM. Dynamics of neurogenesis in the dentate gyrus of adult rats. Neurosci Lett. 2005 Sep;385(1):70-5.
8 Cowen DS, Takase LF, Fornal CA, Jacobs BL. Age-dependent decline in hippocampal neurogenesis is not altered by chronic treatment with fluoxetine. Brain Res. 2008 Sep;1228:149.

9 Hodes GE, Yang L, Van Kooy J, Santollo J, Shors TJ. Prozac during puberty: distinctive effects on neurogenesis as a function of age and sex. Neuroscience. 2009 Oct;163(2):60917.

10 Ho A, Villacis AJ, Svirsky SE, Foilb AR, Romeo RD. The pubertal-related decline in cellular proliferation and neurogenesis in the dentate gyrus of male rats is independent of the pubertal rise in gonadal hormones. Dev Neurobiol. 2012 May;72(5): 743-52.

11 Shome A, Sultana R, Siddiqui A, Romeo RD. Adolescent changes in cellular proliferation in the dentate gyrus of male and female C57BL/6N mice are resilient to chronic oral corticosterone treatments. Front Behav Neurosci. 2018 Aug; 12:192.

12 Yurgelun-Todd D. Emotional and cognitive changes during adolescence. Curr Opin Neurobiol. 2007 Apr;17(2):251-7.

13 Mahmoud R, Wainwright SR, Galea LA. Sex hormones and adult hippocampal neurogenesis: Regulation, implications, and potential mechanisms. Front Neuroendocrinol. 2016 Apr;41:129-52.

14 Tanapat P, Hastings NB, Reeves AJ, Gould E. Estrogen stimulates a transient increase in the number of new neurons in the dentate gyrus of the adult female rat. J Neurosci. 1999 Jul; 19(14):5792-801.

15 Galea LA, Wainwright SR, Roes MM, DuarteGuterman P, Chow C, Hamson DK. Sex, hormones and neurogenesis in the hippocampus: hormonal modulation of neurogenesis and potential functional implications. J Neuroendocrinol. 2013 Nov;25(11):1039-61.

16 Tanapat P, Hastings NB, Gould E. Ovarian steroids influence cell proliferation in the dentate gyrus of the adult female rat in a doseand time-dependent manner. J Comp Neurol. 2005 Jan;481(3):252-65.

17 Spritzer MD, Galea LA. Testosterone and dihydrotestosterone, but not estradiol, enhance survival of new hippocampal neurons in adult male rats. Dev Neurobiol. 2007 Sep;67(10): 1321-33.

18 Schulz KM, Sisk CL. The organizing actions of adolescent gonadal steroid hormones on brain and behavioral development. Neurosci Biobehav Rev. 2016 Nov;70:148-58.

19 Zhang JM, Konkle AT, Zup SL, McCarthy MM. Impact of sex and hormones on new cells in the developing rat hippocampus: a novel source of sex dimorphism? Eur J Neurosci. $2008 \mathrm{Feb} ; 27(4): 791-800$.

20 Bowers JM, Waddell J, McCarthy MM. A developmental sex difference in hippocampal neurogenesis is mediated by endogenous oestradiol. Biol Sex Differ. 2010 Nov; $1(1): 8$. 
21 Goddings AL, Mills KL, Clasen LS, Giedd JN, Viner RM, Blakemore SJ. The influence of puberty on subcortical brain development. Neuroimage. 2014 Mar;88:242-51.

22 Herting MM, Gautam P, Spielberg JM, Kan E, Dahl RE, Sowell ER. The role of testosterone and estradiol in brain volume changes across adolescence: a longitudinal structural MRI study. Hum Brain Mapp. 2014 Nov;35(11): 5633-45.

23 Raznahan A, Shaw PW, Lerch JP, Clasen LS, Greenstein D, Berman R, et al. Longitudinal four-dimensional mapping of subcortical anatomy in human development. Proc Natl Acad Sci USA. 2014 Jan;111(4):1592-7.

24 Paxinos G, Watson C. The rat brain in stereotaxic coordinates. 5th ed. New York: Elsevier Academic Press; 2005.

25 Murty VP, Calabro F, Luna B. The role of experience in adolescent cognitive development: integration of executive, memory, and mesolimbic systems. Neurosci Biobehav Rev. 2016 Nov;70:46-58.

26 Romeo RD. The metamorphosis of adolescent hormonal stress reactivity: A focus on animal models. Front Neuroendocrinol. 2018 Apr;49:43-51.

27 Crews FT, Mdzinarishvili A, Kim D, He J, Nixon K. Neurogenesis in adolescent brain is potently inhibited by ethanol. Neuroscience. 2006;137(2):437-45.

28 Schwarz JM, Sholar PW, Bilbo SD. Sex differences in microglial colonization of the developing rat brain. J Neurochem. 2012 Mar; 120(6):948-63.

29 Day JR, Laping NJ, Lampert-Etchells M, Brown SA, O'Callaghan JP, McNeill TH, et al. Gonadal steroids regulate the expression of glial fibrillary acidic protein in the adult male rat hippocampus. Neuroscience. 1993 Jul; 55(2):435-43.

30 Luquin S, Naftolin F, Garcia-Segura LM. Natural fluctuation and gonadal hormone regulation of astrocyte immunoreactivity in dentate gyrus. J Neurobiol. 1993 Jul;24(7):91324.

31 Brummelte S, Galea LA. Chronic high corticosterone reduces neurogenesis in the dentate gyrus of adult male and female rats. Neuroscience. 2010 Jul;168(3):680-90.
32 Isgor C, Kabbaj M, Akil H, Watson SJ. Delayed effects of chronic variable stress during peripubertal-juvenile period on hippocampal morphology and on cognitive and stress axis functions in rats. Hippocampus. 2004;14(5): 636-48.

33 Rahimi O, Claiborne BJ. Morphological development and maturation of granule neuron dendrites in the rat dentate gyrus. Prog Brain Res. 2007;163:167-81.

34 Koss WA, Frick KM. Sex differences in hippocampal function. J Neurosci Res. 2017 Jan; 95(1-2):539-62.

35 Deng W, Aimone JB, Gage FH. New neurons and new memories: how does adult hippocampal neurogenesis affect learning and memory? Nat Rev Neurosci. 2010 May;11(5): 339-50.

36 Aimone JB, Li Y, Lee SW, Clemenson GD, Deng W, Gage FH. Regulation and function of adult neurogenesis: from genes to cognition. Physiol Rev. 2014 Oct;94(4):991-1026.

37 Levone BR, Cryan JF, O'Leary OF. Role of adult hippocampal neurogenesis in stress resilience. Neurobiol Stress. 2014 Nov;1:147-55. 